\title{
Persistent attenuation and enhancement of the earthworm main muscle contraction generator response induced by repeated stimulation of a peripheral neuron
}

Y.C. Chang, E.C.L. Caffaro, Z. Assmé and A.B. Bartoszeck
Departamento de Fisiologia, U niversidade Federal do Paraná, Curitiba, PR, Brasil

\section{Correspondence}

Y.C. Chang

Departamento de Fisiologia

Setor de Ciências Biológicas, UFPR

Caixa Postal 8621

80011-970 Curitiba, PR

Brasil

Fax: +55-41-266-2042

E-mail: changyc@cce.ufpr.br

Part of a Master's thesis presented by E.C.L. Caffaro to the Departamento de Fisiologia, UFPR, Curitiba, PR, Brasil.

Received January 20, 1997 Accepted July 27, 1998

\section{Abstract}

Responses evoked in the earthworm, Amynthas hawayanus, main muscle contraction generator M-2 (postsynaptic mechanical-stimulus-sensitive) neuron by threshold mechanical stimuli in 2-s intertrial intervals (ITI) were used as the control or unconditioned responses (UR). Their attenuation induced by decreasing these intervals in nonassociative conditioning and their enhancement induced by associating the unconditioned stimuli (US) to a train of short ( $0.1 \mathrm{~s}$ ) hyperpolarizing electrical substitutive conditioning stimuli (SCS) in the Peri-Kästchen $(\mathrm{PK})$ neuron were measured in four parameters, i.e., peak numbers $(\mathrm{N})$ and amplitude $(\overline{\mathrm{AMP}})$ averaged from 120 responses, sum of these amplitudes $\left(\Sigma_{A M P}\right)$ and the highest peak amplitude (V) over a period of $4 \mathrm{~min}$. Persistent attenuation similar to habituation was induced by decreasing the control ITI to $0.5 \mathrm{~s}$ and $2.0 \mathrm{~s}$ in nonassociative conditioning within less than 4 min. Dishabituation was induced by randomly pairing one of these habituated US to an electrical stimulus in the PK neuron. All four parameters of the UR were enhanced by forward (SCS-US), but not backward (US-SCS), association of the US with 25,100 and $250-\mathrm{Hz}$ trains of SCS with $40-\mathrm{ms}$ interstimulus intervals (ISI) for $4 \mathrm{~min}$ and persisted for another $4 \mathrm{~min}$ after turning off the SCS. The enhancement of these parameters was proportional to the SCS frequencies in the train. No UR was evoked by the SCS when the US was turned off after 4 min of classical conditioning.

\section{Introduction}

The purpose of the present article, third in this series $(1,2)$, is to demonstrate that parameters such as peak number and amplitude of a response evoked in the earthworm, Amynthas hawayanus, neuron can be modified in a paradigm comparable to that of conditioning experiments of neuronal re-

\section{Key words}

- Persistent attenuation and enhancement

- Non-associative conditioning

- Associative conditioning

- Earthworm neuron response parameters sponses in other animals (3-6) and in intact earthworms $(7,8)$. By conditioning, we mean that these modifications induced in the response parameters will persist after cessation of their induction. The enhancement reported in the second article (2) in this series was induced by a simple facilitation but not by conditioning because it did not persist. A different method of induction with 
Figure 1 - Experimental setup and control response. EMNC Epidermis-muscle-nerve-cord preparation with sectioned left third nerve and with the epidermis-muscle piece partitioned into two lateral columns. The central column is the nerve cord with three pairs of segmental nerves, anterior end upward. US, Mechanical stimulus to the setal shaft (S) on the epidermal surface of the left epidermis-muscle piece. The square symbol at top right is the mechano-electrical transducer for recording muscle contraction (C). M-2, Response recorded from the $M-2$ neuron after section of the left third nerve; $\mathrm{E}$, effector muscle electrical response; PK, Peri-Kästchen without response after sectioning; SCS, substitutive conditioning stimulus in the form of a hyperpolarizing short electrical pulse train to the PK neuron (see explanation in text). Calibration: $\mathrm{C}$, in $5 \mathrm{~g}$ and $5 \mathrm{~s} . \mathrm{M}-2$, E and $\mathrm{PK}$, in $50 \mathrm{mV}$ and $50 \mathrm{~ms}$. SCS, schematic, no calibration.

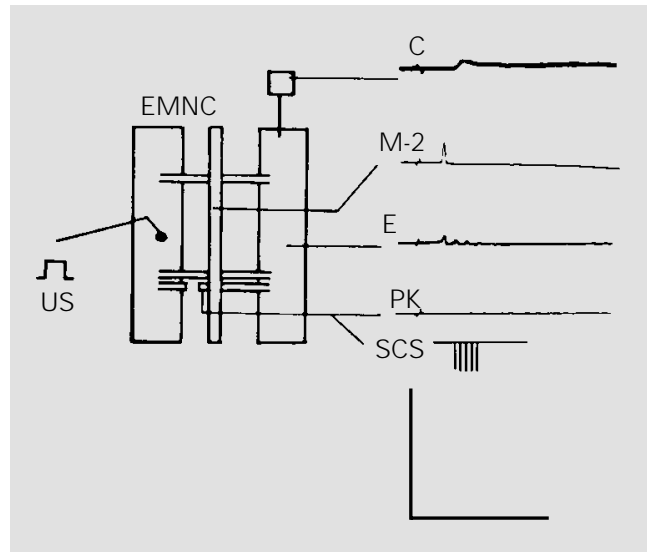

the same stimuli $(1,2)$ will be used to induce persistent postsynaptic modifications in the form of attenuation and enhancement in the present article. The response evoked in the M-2 (postsynaptic mechanical-stimulus-sensitive) neuron (1) was used as the control or unconditioned response (UR) to show these modifications in the present article. An electrical intracellular stimulus to the PeriKästchen (PK) neuron was used as the substitutive conditioning stimulus (SCS) similar to that used in the second article (2) but with modified parameters. The non-associative conditioning with only the unconditioned stimuli (US) and the associative conditioning with US to the M-2 and SCS to the PK neurons were tested. Different combinations of these two stimuli, such as forward (SCSUS) and backward (US-SCS) associations, classical conditioning after cessation of the US and potentiation after cessation of the SCS, were tested.

\section{Material and Methods}

The preparations and experimental procedures used in this article were the same as shown in Figure 2-2 of the second article (2) in this series. The main muscle contraction generator M-2 (postsynaptic mechanicalstimulus-sensitive) neuron response (1) evoked by a threshold mechanical stimulus (US) (Figure 1) was used to indicate the induced postsynaptic excitability modifica- tions, in the control or the UR. The bifurcated conditioning response (CR) evoked in the PK neuron by this mechanical stimulus (2) was eliminated by sectioning the left third nerve (Figure 1). An electrical stimulus was delivered to the PK neuron as the SCS (Figure 1). An action potential was evoked in the PK neuron by a threshold (4 nA) SCS in Figure 3-3 of the second article (2). In order not to confuse this action potential with the UR or with the alpha-response (8), the SCS in the present article must not evoke any response in the PK neuron. Such an SCS was found by breaking the long electrical pulse $(50.0 \mathrm{~ms})$ used in the last article (2) into a train of the same duration $(50.0 \mathrm{~ms})$ but consisting of short $(0.1 \mathrm{~ms})$ electrical pulses (SCS, Figure 1 and SCS, Figure 4-3). The amplitude of the SCS was maintained at the threshold value of $4 \mathrm{nA}$ in the present study although it did not evoke any response in the PK neuron or in the M-2 neuron even by a superthreshold amplitude of more than $10.0 \mathrm{nA}$ in either polarity. Further advantages of using this new SCS are described in the next paragraph. The US parameters, i.e., peak numbers and amplitude, must be maintained constant throughout the duration of the control experiment. The first parameter, the UR peak number, was always constant as $1.0 \pm 0.0$ (Figure 1) after sectioning the left third nerve (2). This number changed only in experiments of induced modifications. The other parameter, the amplitude (AMP) of these UR peaks, was not always constant when evoked by repeated US in constant intertrial intervals (ITI). A long ITI which evoked nearly constant UR amplitude was used in control experiments (Figure 2). As these UR amplitudes were never exactly constant (see Results), they were measured by three parameters, the average amplitudes, $\overline{\mathrm{AMP}}$, the sum of all amplitudes, $\Sigma_{A M P}$, and the highest peak amplitude (V) ever recorded in $12 \mathrm{~min}$ by repeated US in constant ITI. The reason for limiting these experiments to 12-min sessions but not any longer was the 
mechanical instability caused by frequent wiggling movements of the live preparation. Data from more than 100 experiments on 45 of the 61 preparations were rendered useless due to multiple entries of microelectrodes into the wrong neurons when their Lucifer Yellow-CH-filled images were examined. Mechanical restraining of the preparations was ineffective because these movements could occur in very small areas and was undesirable because it acted as another mechanical stimulus. Anesthetics, such as triacaine and low temperature, attenuated the electrical response and muscle contraction. Neuromuscular blocking agents, such as $\mathrm{Mg}^{2+}$ and curare, did not stop this movement because they did not block the transmission between muscle cell synapses nor did they stop the spontaneous excitation of the muscle cell membrane (9). The longest time the microelectrodes could manage to stay in the same neuron without straying was $13.5 \pm 2.4 \mathrm{~min}$, or $810 \pm 144 \mathrm{~s}$, in only 16 of these unrestrained preparations in normal physiological solution. This time was divided into three equal sessions of $4 \mathrm{~min}$, or $240 \mathrm{~s}$, each. The first 4-min session was used to record the M-2 neuron responses as the control UR evoked by the US alone (Figure 2 and Tables 1 and 2). The second 4-min session was used to induce the modifications in these UR by either the non-associative (Figure 3 and Table 1) or the associative (Figures 4, 5 and Table 2) method. The third and last 4-min session was used to record the maintenance of the persistent UR parameter enhancements induced in the second session (Figures 4, 5 and Table 2).

In order to induce persistent, instead of instantaneous (2), modifications in the UR as required for a conditioning paradigm, the US and SCS used in the present study were different from those used in the two preceding studies $(1,2)$. The US alone was used for the non-associative paradigm. When these US were delivered at short ITI, their cumulative effects manifested as attenuation of the
UR parameters. Constant UR parameters could be evoked by long ITI, such as $2 \mathrm{~s}$, or $0.5-\mathrm{Hz}$ frequency, in control experiments (Figure 2). Attenuation, or habituation, of the UR parameters was induced by decreasing these ITI to half a second $(0.2 \mathrm{~Hz})$ and to $0.2 \mathrm{~s}(0.5 \mathrm{~Hz})$ in the non-associative conditioning (Figure 3 and Table 1). Dishabituation of the habituated UR was induced by randomly pairing one of the US to an electrical stimulus in the PK neuron (DH; Figure 3-2). Persistent enhancement of UR parameters was induced gradually over 4 min by associating the US in the M-2 neuron with the train of short-pulsed SCS in the PK neuron through two microelectrodes coupled to the Dagan 8500 high impedance bridge with measurable constant interstimuli intervals (ISI). These ISI could be changed in experiments of forward (SCS-US) and backward (USSCS) associations by a built-in synchronizer in the CURITIBA-1 stimulator (manufactured by the authors of this article, Curitiba, PR, Brazil). Maintenance of persistent UR parameter enhancements was demonstrated by turning off the US but leaving the SCS on in the last 4-min session of classical conditioning and by turning off the SCS but leaving the US on in the last 4-min session of potentiation (Figures 4, 5 and Table 2).

\section{Results}

\section{Control response parameters}

Four parameters were measured from the control responses (UR) evoked in the M-2 neuron by threshold $(3.0 \mathrm{~V}, 5 \mathrm{~ms})$ mechanical stimuli (US; Figure 1) in 2.0-s ITI, or 0.5$\mathrm{Hz}$ frequency. The first parameter, the UR peak number $(\mathrm{N})$, was always constant as 1.0 \pm 0.0 after sectioning the left third nerve (M2 ; Figure 1). It varied only during further experiments. The UR peak AMP varied in $0.81 \pm 0.12$-min, or $48.6 \pm 7.2$-s periodic cycles from 0 (no response) to more than $10.0 \mathrm{mV}$ in each individual preparation over 
Figure 2 - Control response amplitude variations. An example of control response amplitude variations in one of the 16 preparations. Notice the five complete cycles within $4 \mathrm{~min}$. The intertrial interval between succeeding responses was $2 \mathrm{~s}$, or US $\mathrm{f}=$ $0.5 \mathrm{~Hz}$. The average amplitude, $\overline{\text { AMP, }}$ of these 120 responses (including zero) was $1.70 \pm 0.82$ $\mathrm{mV}$. Its high SEM was caused by the wide variation range from $12 \mathrm{mV}$ to $0 \mathrm{mV}$. Their total sum, $\Sigma_{\text {AMP, was }} 198 \mathrm{mV}$. The highest peak (between 3 and $4 \mathrm{~min}$ ) was $12 \mathrm{mV}$. These parameter values were averaged again among 16 preparations in Tables 1 and 2 and were compared to the induced modifications in Figures 3, 4 and 5. UR, Unconditioned responses. the entire experimental session, such as the example shown in Figure 2. They were constant as $1.81 \pm 0.26 \mathrm{mV}$ when averaged from 16 preparations ( $\overline{\mathrm{AMP}}$ ) (Tables 1 and 2 ). This $\overline{\text { AMP }}$ was adequate in describing the

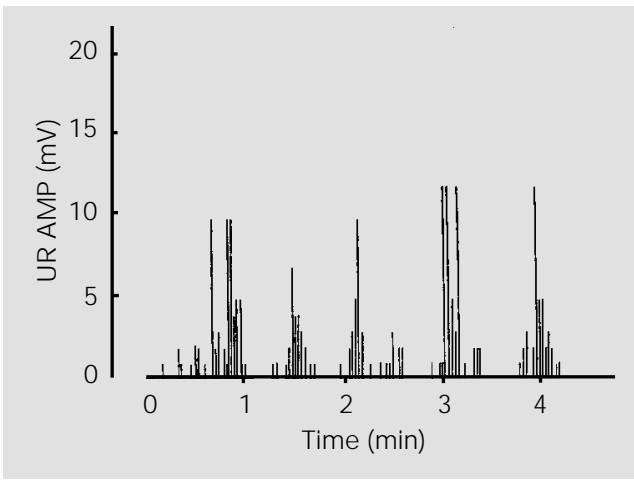

Table 1 - Attenuation of unconditioning response (UR) parameters induced by non-associative conditioning.

US, Unconditioning stimulus. $\overline{\mathrm{AMP}}$, Average amplitude in $\mathrm{mV} ; \Sigma_{\mathrm{AMP}}$, amplitude sum in $\mathrm{mV}$; $\mathrm{V}$, highest peak in $\mathrm{mV}$.

\begin{tabular}{lccc}
\hline UR & \multicolumn{3}{c}{ US } \\
\cline { 2 - 4 } & $\begin{array}{c}0.5 \mathrm{~Hz} \\
(\text { Control })\end{array}$ & $2.0 \mathrm{~Hz}$ & $5.0 \mathrm{~Hz}$ \\
& & \\
\hline$\overline{\mathrm{AMP}}$ & $1.81 \pm 0.26$ & $1.04 \pm 0.16$ & $0.68 \pm 0.15$ \\
$\Sigma_{\text {AMP }}$ & $234 \pm 63$ & $125 \pm 41$ & $79 \pm 31$ \\
$\mathrm{~V}$ & $12.5 \pm 1.7$ & $10.5 \pm 2.3$ & $9.6 \pm 3.3$
\end{tabular}

overall amplitude variations induced in the experiments but was not sensitive in showing the presence and absence of individual peaks. Also, it obliterated the highest peak amplitude by averaging. Two more parameters were measured from these amplitudes to compensate for the insufficiencies of $\overline{\mathrm{AMP}}$. They were the $\Sigma_{A M P}$ and $\mathrm{V}$ recorded in each session. Both of them were averaged again among the preparations. The values of these four parameters are listed in Tables 1 and 2.

\section{Persistent UR attenuation induced by non- associative conditioning}

The UR parameters were attenuated when they were evoked by constant US in ITI shorter than that of the control, such as $0.5 \mathrm{~s}$, or $2.0 \mathrm{~Hz}$ (Figure 3-1), and $0.2 \mathrm{~s}$, or $5.0 \mathrm{~Hz}$ (Figure 3-2), in the second 4-min session of non-associative conditioning. $\mathrm{N}$ became zero within a few seconds, as also did the effector muscle contraction. The average decreases of $\overline{\mathrm{AMP}}, \Sigma_{A M P}$ and $\mathrm{V}$ in five preparations were compared to their values evoked by control ITI of $2.0 \mathrm{~s}$, or $0.5 \mathrm{~Hz}$, in Figure 3-3, 3-4 and 3-5. The decrease of V (Figure 3-5) was less apparent than the decrease of the other two parameters because it was measured at the beginning of each experiment before it began to be attenuated. This attenu-

Table 2 - Persistent enhancement of unconditioning response (UR) parameters induced by associative conditioning.

All $\overline{A M P}, \Sigma_{\mathrm{AMP}}$ and $\mathrm{V}$ were averaged from the first peaks obtained within $4 \mathrm{~min}$. Subsequent peaks were counted for N. All ISI between paired SCS-US were $40 \mathrm{~ms}$. All ITI $=2 \mathrm{~s}$. SCS, Substitutive conditioning stimulus. $\mathrm{N}$, Peak number; $\overline{\mathrm{AMP}}$, average amplitude in $\mathrm{mV} ; \Sigma_{\mathrm{AMP}}$, amplitude sum in $\mathrm{mV} ; \mathrm{V}$, highest peak in $\mathrm{mV}$.

\begin{tabular}{|c|c|c|c|c|c|c|c|}
\hline \multirow[t]{3}{*}{ UR } & \multicolumn{7}{|c|}{ SCS } \\
\hline & \multirow{2}{*}{$\begin{array}{c}0 \mathrm{~Hz} \\
\text { Control }\end{array}$} & \multicolumn{2}{|c|}{$25 \mathrm{~Hz}$} & \multicolumn{2}{|c|}{$100 \mathrm{~Hz}$} & \multicolumn{2}{|c|}{$250 \mathrm{~Hz}$} \\
\hline & & Induced & Persisted & Induced & Persisted & Induced & Persisted \\
\hline $\mathrm{N}$ & $1.0 \pm 0.0$ & $1.6 \pm 0.9$ & $1.0 \pm 0.0$ & $14.5 \pm 2.7$ & $9.8 \pm 3.2$ & $25.3 \pm 4.5$ & $16.5 \pm 5.5$ \\
\hline $\begin{array}{l}\overline{\mathrm{AMP}} \\
\Sigma_{\mathrm{AMP}}\end{array}$ & $\begin{aligned} 1.81 & \pm 0.26 \\
202 & \pm 56\end{aligned}$ & $\begin{array}{c}3.3 \pm 0.91 \\
437 \pm 87\end{array}$ & $\begin{array}{c}2.50 \pm 0.61 \\
330 \pm 63\end{array}$ & $\begin{array}{r}5.64 \pm 1.18 \\
641 \pm 14.9\end{array}$ & $\begin{array}{c}4.05 \pm 0.95 \\
539 \pm 152\end{array}$ & $\begin{array}{c}16.6 \pm 3.4 \\
1976 \pm 282\end{array}$ & $\begin{array}{c}6.52 \pm 1.13 \\
1005 \pm 194\end{array}$ \\
\hline $\mathrm{V}$ & $12.5 \pm 1.7$ & $15.2 \pm 2.7$ & $11.1 \pm 0.7$ & $30.5 \pm 4.2$ & $14.8 \pm 1.3$ & $41.8 \pm 6.1$ & $25.6 \pm 2.8$ \\
\hline
\end{tabular}




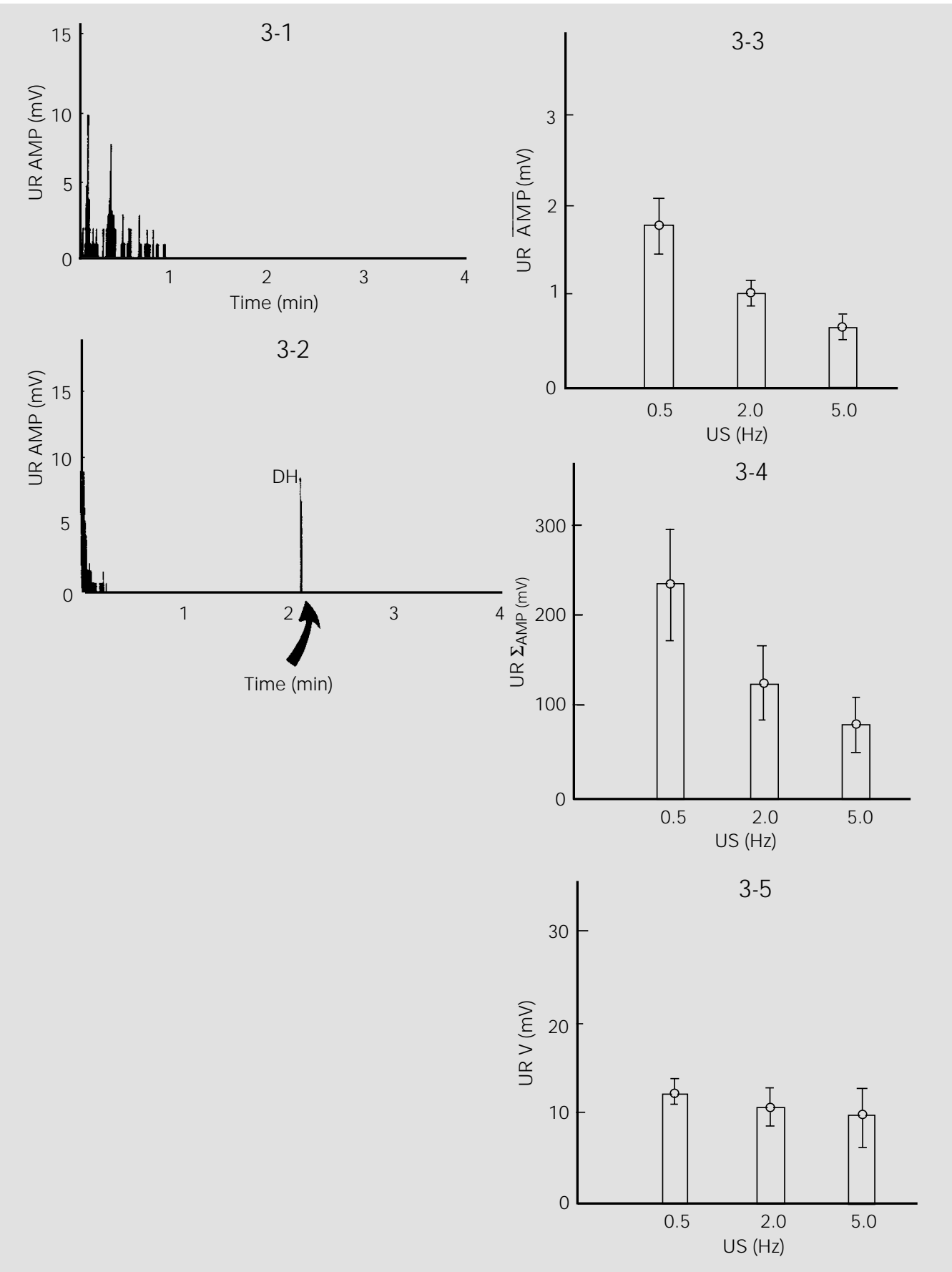

ation, or non-response, did not recover in the third 4-min session (not shown in Figure 3) even if the ITI was lengthened to $1 \mathrm{~min}$, or 17 $\mathrm{x} 10^{-3} \mathrm{~Hz}$. To test if these attenuations were actually caused by a mechanism similar to habituation instead of nonspecific damage to these preparations, a long depolarizing electrical stimulus similar to that delivered to the PK neuron in the second study (2) was coupled randomly to this mechanical US at a time when there was hardly any response (arrow; Figure 3-2). A couple of UR were induced at their highest values (DH; Figure 3-2). This experiment justifies the term habitu-
Figure 3 - Persistent UR parameter attenuation induced by nonassociative conditioning.

3-1, Example of unconditioned response (UR) amplitude attenuation induced by decreasing the unconditioned stimulus (US) intertrial interval (ITI) to $0.5 \mathrm{~s}$, or $\mathrm{f}$ $=2.0 \mathrm{~Hz}$.

3-2, Example of UR amplitude attenuation induced by decreasing the US ITI to $0.2 \mathrm{~s}$, or $\mathrm{f}=5.0$ $\mathrm{Hz}$. DH, Dishabituation induced by pairing one of the US to an intracellular electrical stimulus in the PK neuron (see text). Notice that its effect lasted for two subsequent responses.

3-3, Comparison of UR AMP induced by US $f=2.0 \mathrm{~Hz}$ and $f=$ $5.0 \mathrm{~Hz}$ to that induced by the control $\mathrm{f}=0.5 \mathrm{~Hz}$. $\mathrm{N}=120$.

3-4, Comparison of UR $\Sigma_{\text {AMP }}$ induced by US $\mathrm{f}=2.0 \mathrm{~Hz}$ and $\mathrm{f}=$ $5.0 \mathrm{~Hz}$ to that induced by the control $\mathrm{f}=0.5 \mathrm{~Hz}$. N $=120$.

3-5, Comparison of UR V induced by US $\mathrm{f}=2.0 \mathrm{~Hz}$ and by $\mathrm{f}$ $=5.0 \mathrm{~Hz}$ to that induced by the control $\mathrm{f}=0.5 \mathrm{~Hz}$. $\mathrm{N}=120$. See Table 1 for their actual values. $\mathrm{V}$ Highest peak amplitude. 
ation and dishabituation used in this article.

\section{Persistent UR enhancement induced by associative conditioning}

Enhancement of all four parameters (including the peak number, N) of UR was induced by forwardly associating the US with the SCS (SCS-US) in 9 of these 16 preparations for 4-min. Instead of an instantaneous enhancement such as that induced in the second article (2) of this series, the enhancement induced in this section was gradual during the second 4-min session (Figure 4). Instead of coinciding, the SCS and US must be separated by a measurable interstimulus interval (ISI) in this experiment (Figure 4-3, inset). The most efficient ISI in inducing UR enhancement was found to be nearly $40 \mathrm{~ms}$. Although both depolarizing and hyperpolarizing SCS induced UR enhancements, the hyperpolarizing SCS induced higher UR values than did the depolarizing SCS. Examples of enhancement of all four parameters induced by hyperpolariz- ing SCS separated by $40 \mathrm{~ms}$ ISI with respect to the US in the second 4-min sessions are shown in Figure 4. These enhancements were found to be proportional to the train frequencies in the SCS. Enhancements induced by 25,100 and $250-\mathrm{Hz}$ SCS are shown in one example in Figure 4 and were averaged from nine preparations in Figure 5. SCS frequencies higher than $250 \mathrm{~Hz}$, such as $500 \mathrm{~Hz}$, failed to induce any change in the UR. It seemed that an upper limit of frequency response was reached beyond $250 \mathrm{~Hz}$. This preparation was unable to distinguish the individual pulses in a train at this frequency from a long continuous pulse which did not induce any change in the UR if separated by an ISI (2). After turning off the SCS at the end of the second 4-min session while leaving the US on, enhancements of all four parameters did not return to their control values immediately but declined (Figure 4) gradually during the third 4-min session (Figures 4 and 5). The effector muscle contraction (C; inset, Figure 4-3) was also enhanced both before and after turning off the SCS but
Figure 4 - Examples of persistent UR parameter enhancements induced and maintained by associative conditioning. 4-1, Unconditioning response (UR) amplitude enhancement in one of the preparations induced by $25-\mathrm{Hz}$ substitutive conditioning stimulus (SCS) and maintained after SCS off.

4-2, Induced by $100 \mathrm{~Hz}$ SCS on and maintained after SCS off. 4-3, Induced by $250 \mathrm{~Hz}$ SCS on and maintained after SCS off. Inset, Example records taken after SCS on and SCS off in Figure 43. C, Effector muscle contraction.

Calibration: $5 \mathrm{~g}$ and $5 \mathrm{~s}$. M-2 response with SCS, unconditioning stimulus (US) and interstimulus interval (ISI). Calibration: 50 $\mathrm{mV}$ and $50 \mathrm{~ms}$.
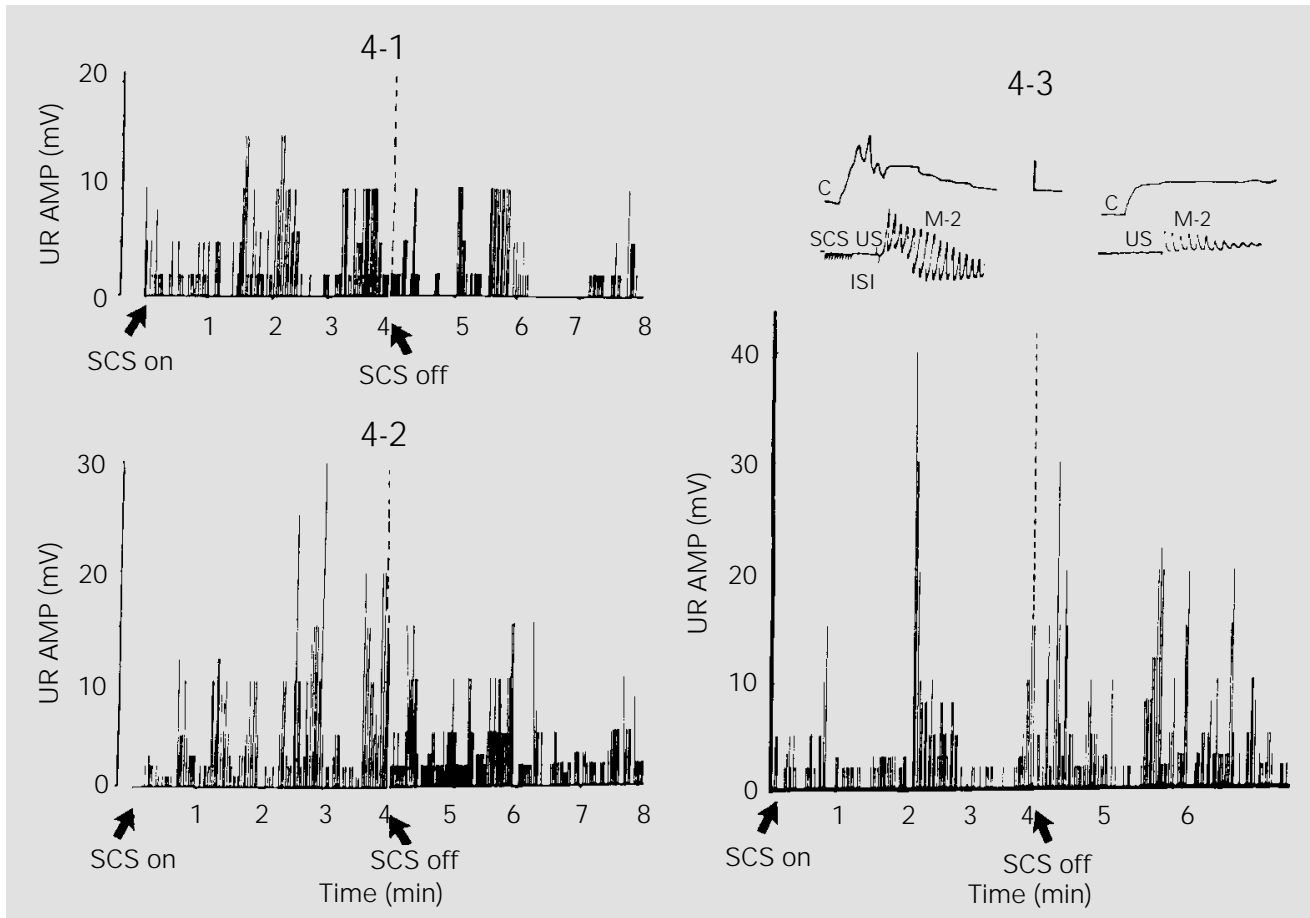
was not measured as a parameter due to its irregular time course.

Backward association (US-SCS) did not induce any observable change in the UR because the SCS were always superimposed on the UR and because these SCS did not evoke any response in the M- 2 neuron by themselves. This rationale may explain why classical conditioning did not work in these
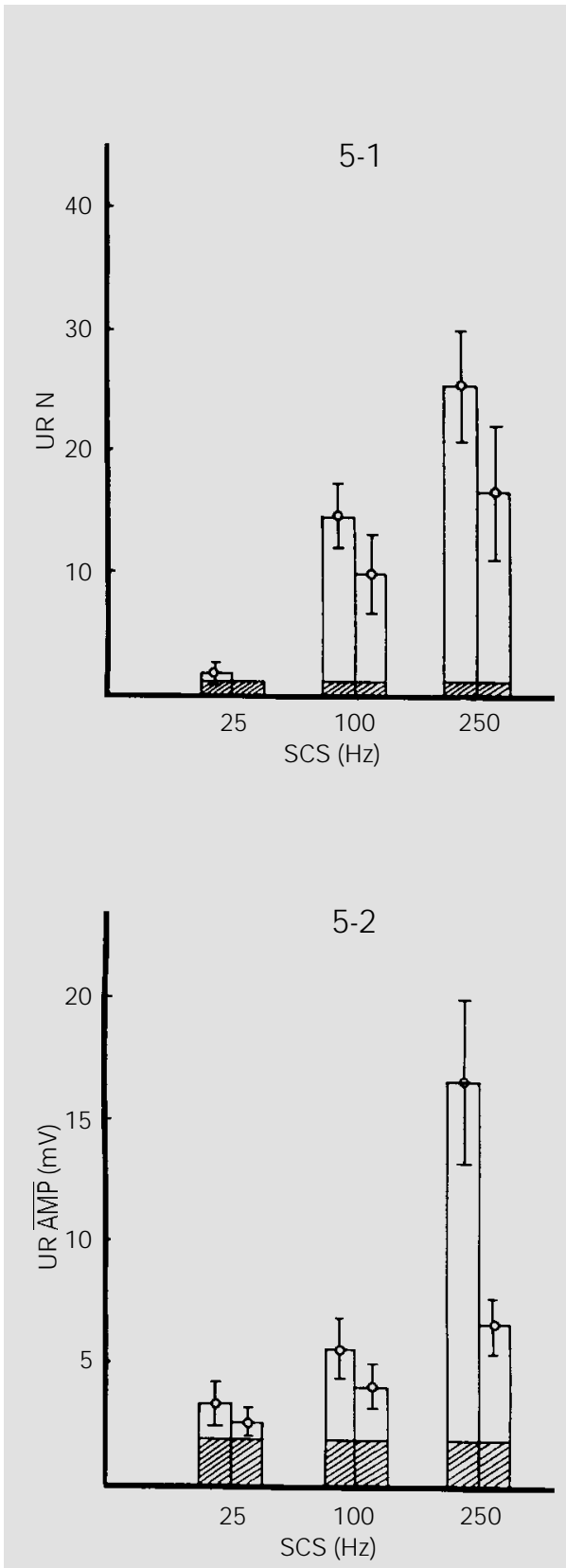

experiments. No response was evoked if the US was turned off while leaving the SCS on after 4 min of forward, or backward, association.

\section{Discussion}

Although both attenuation and enhancement induced in this preparation resembled

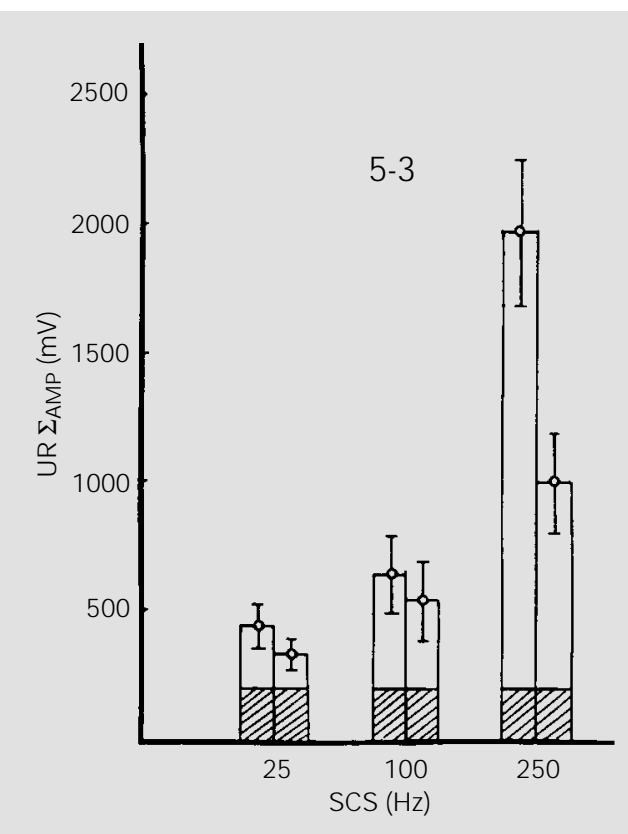

Figure 5 - Induction and maintenance of persistent unconditioning response (UR) parameter enhancements by associative conditioning.

5-1, Peak number (N) of enhancements induced (first column in each pair of histographs) by $25-, 100-$ and $250-\mathrm{Hz}$ SCS maintained (second column in each pair of histographs) after their cessation. The shaded parts under each histograph are the control values before their induction.

5-2, $\overline{\mathrm{AMP}}$ enhancements. $5-3, \Sigma_{\text {AMP }}$ enhancements. 5-4, Highest peak amplitude (V) enhancements. SCS, Substitutive conditioning stimulus. 
those induced in the other animals (3-6) and in other species of intact earthworms $(7,8)$, basic differences in their control responses must first be considered. The most conspicuous peculiarity noticed in the control response of this preparation was the periodic variation (Figure 2) of their peak amplitudes. This periodic variation made it necessary to measure four different aspects of this control response for comparison instead of measuring a single parameter alone. As a graded response (9), the amplitude of an intracellular response of this preparation was expected to vary with the variations of stimulus intensity. The intensity of mechanical stimuli (US; Figure 1) which evoked this control response was held constant in all experiments. Therefore, this periodic variation must have been caused by intensity variation in circuits between the primary afferent neuron and the generator M-circuit neurons where these responses were recorded. Hypothetical P-circuits were proposed in Figure 1 of this article and in a previous article (10). One of them is supposed to determine (2) the variations in $\mathrm{M}-2$ response peak number $(\mathrm{N})$ shown in Figures 4 and 5 of this article. Others may determine the variations in $\mathrm{M}-2$ response amplitude through functions similar to that of the leech swimming rhythm oscillator circuit (11). Both kinds of circuits may be facilitated by the conditioning stimuli and also by the SCS in the PK neuron.

Important differences were found when comparing these results to those of the above mentioned animals. Classical conditioning, i.e., the UR evoked by conditioning stimuli after the US were turned off, was readily demonstrated in the intact earthworms $(7,8)$ but was not demonstrated in the reduced preparation of the present study. Potentia- tions were induced by both forward and backward associations in Aplysia gill-withdrawal response $(3,4)$ but were induced only by forward association in this preparation. The so-called potentiation persisted only for less than 4-min in this preparation while it persisted for hours in Aplysia. There is no explanation as yet for these differences.

The train of short pulses used as SCS in the present study seemed to function differently from the long pulses in the second study (2) in this series. A depolarizing long pulse evoked an action potential in the PK neuron and induced instantaneous enhancement in the M-2 neuron response while a hyperpolarizing long pulse inhibited these events. It was the hyperpolarizing short pulse which induced higher persistent enhancement in the M-2 neuron response than that induced by the depolarizing short pulse. No response was evoked in the PK or in the M2 neuron by short pulses of both polarities. There is not yet a satisfactory explanation for these seemingly opposite functions for two pulses of the same polarity but differing only in duration. A speculative explanation was based on the observation that earthworm neuron, as well as muscle, membranes seemed to respond better to the depolarization of a repolarizing phase in a hyperpolarizing pulse (Figure 3 in Ref. 1) than to a depolarizing phase in a depolarizing pulse. Thus, the membrane excitability was actually determined by the duration of hyperpolarization before the rebounding (repolarization). This explains why the same repolarization of a short hyperpolarizing pulse evoked no response but the cumulative effects of many such short hyperpolarizations manifested themselves as a potentiation in the M-2 neuron response. 


\section{References}

1. Chang YC, Assmé Z, Caffaro ECL \& Bartoszeck AB (1998). Identification of the main generator source of longitudinal muscle contraction in the earthworm ventral nerve cord. Brazilian J ournal of Medical and Biological Research, 31: 12851294.

2. Chang $Y C$, Assmé $Z \&$ Bartoszeck $A B$ (1998). Facilitation of the main generator source of earthworm muscle contraction by a peripheral neuron. Brazilian J ournal of Medical and Biological Research, 31: 1295-1302.

3. Kandel ER \& SchwartzJ H (1982). Molecular biology of learning: Modulation of transmitter release. Science, 218: 433443.

4. Walters ET \& Byrne J H (1985). Long-term enhancement produced by activity de- pendent modulation of Aplysia sensory neurons. J ournal of Neuroscience, 5: 662672.

5. Sastry BR, Goh J W \& Auyeung A (1986). Associative induction of posttetanic and long-term potentiations in CAI neurons of rat hippocampus. Science, 232: 988-990.

6. Artola A \& Singer W (1993). Long-term depression of excitatory synaptic transmission and its relationship to long-term potentiation. Trends in Neurosciences, 16: 480-487.

7. Ratner SC \& Miller KR (1959). Classical conditioning in earthworms, Lumbricus terrestris. J ournal of Comparative and Physiological Psychology, 52: 102-105.

8. Abramson $\mathrm{Cl} \&$ Buckbee DA (1995). Pseudoconditioning in earthworms (Lumbricus terrestris): Support for nonassocia- tive explanation of classical conditioning phenomena through an olfactory paradigm. J oumal of Comparative Psychology, 109: 390-393.

9. Chang YC (1975). The endplate and graded potentials from the neuromuscular system of the earthworm, Pheretima hawayana, R. Comparative Biochemistry and Physiology, Section A, 51: 237-240.

10. Assmé $Z \&$ Chang YC (1990). Decremental propagation of reflex spikes along giant axons of the earthworm, Amynthas hawayanus. Brazilian J ournal of Medical and Biological Research, 23: 329-332.

11. Friesen WO, Poon M \& Stent GS (1976). An oscillatory neuronal circuit generating a locomotory rhythm. Proceedings of the National Academy of Sciences, USA, 73: 3734-3738. 